Proceedings of the 2011 Winter Simulation Conference

S. Jain, R. R. Creasey, J. Himmelspach, K. P. White, and M. Fu, eds.

\title{
SIMULATION OF WIRELESS SENSOR NETWORKS UNDER PARTIAL COVERAGE
}

\author{
Ruth Lamprecht \\ Peter Kemper \\ College of William and Mary \\ Department of Computer Science \\ Wiiliamsburg, VA 23187 USA
}

\begin{abstract}
This paper presents research using simulation to explore the sensitivity of the network lifetime of a wireless sensor network (WSN) under the constraint to maintain a chosen coverage percentage when different aspects of the node model are included. Specifically, we begin with a simple sensor node that can transition between an Awake mode and a Sleep mode, dependent on meeting the coverage constraint with a simple battery model that expends energy when the node is in the Awake mode. We then compare this network behavior to when the battery model includes battery recovery behavior. We conclude that while the difference between the behaviors is small, they are significant enough to warrant the inclusion of a more sophisticated battery model when modeling wireless sensor networks.
\end{abstract}

\section{INTRODUCTION}

A wireless sensor network (WSN) is a set of sensor nodes that can be used to monitor some situation. These nodes share data through wireless connections, with the goal being to have the data collected to be analyzed somewhere else. The nodes can be all of the same type, or the network can be a heterogeneous mix of multiple different kinds of nodes. A network can consist of only a few nodes or of several hundred or thousand nodes. A common usage for sensor networks is to do observance of the area to detect activity of some kind. This may be in an environmental capacity to monitor weather and wildfire behavior (Douglas et al. 2006), water quality (Ailamaki et al. 2003), or numerable other natural occurrences. WSNs have also been applied to underwater applications for exploration and data collection (Akyildiz, Pompili, and Melodia 2005). Another use for WSNs is for structural monitoring to detect damage to buildings, bridges and large transports (Xu et al. 2004). Intrusion detection (Arora et al. 2004) is also a common application for WSNs.

Modeling WSNs is a useful practice to decide on the desired set-up of a real system. One can explore parameter setting changes and quickly see the effect on the network behavior and lifetime. But in doing so, a modeler must decide what aspects of the real system to include in the model. Excessive detail can lead to unnecessary work in the analysis. Law and Kelton (2000) provide guidelines for determining the amount of detail to include. The details should directly relate to the specific issues being studied, but more details should be included if they affect the validity or credibility of the model.

The simplest sensor node has two main modes, Awake and Sleep, a protocol for deciding when to switch between modes, and a battery that determines how long a sensor can work. The simplest battery model is a drainage of power over the time that a sensor is in the Awake mode, which can be improved by including battery recovery, which occurs when the node is in the Sleep mode, either at a constant rate or at a variable rate dependent on the rest of the state of the node.

This paper explores the sensitivity of the network lifetime of a detection sensor network to the inclusion of more complex information in a node model. The network constraint used is that a coverage percentage, or the number of sensors in the Awake mode, must be maintained. The focus here is how including different 


\section{Lamprecht and Kemper}

aspects of the node, specifically relating to the battery, into the model affect the outcome of the simulation. The aspects of interest are including, or not, battery recovery, and simple or more complex rules for such recovery. Model exploration is done with the Möbius program (PERFORM 2011). The sensor node model specifics come from Ren et al. (2005) while the more sophisticated battery model comes from Jongerden et al. (2010).

This paper continues as follows: Section 2 discusses related work and the Möbius modeling tool. Section 3 defines the model for a wireless sensor node and network used in this paper. Section 4 presents the simulation study results and Section 5 provides a brief summary of this work.

\section{RELATED WORK}

\subsection{Modeling Wireless Sensor Networks}

Ren et al. (2005) examine the detection probability for different network coverage percentages with varying densities and node activation probabilities. They use their simulation experiments to define and test an analytical model that can be used to test the quality of object detection under different network conditions and scheduling protocols, finding that a random protocol for when a node transitions between the two modes provides the best lifetime. Lu et al. (2009) examine how to maximize network lifetime while maintaining quality of service, as defined by coverage and connectivity. They examine modifying a sensor's transmission and sensing ranges to save energy, under the assumptions that all sensors are connected and any target is within the maximum sensing range of at least one sensor. They find that the more adjustable the ranges are, the longer the network lifetime. Chiasserini and Garetto (2004) propose a sensor network model to examine energy consumption, network capacity and data delivery. They verify their model through both numerical analysis and simulation. One finding from their research is that as the data delivery delay decreases, the energy consumption increases. Cao et al. (2005) propose a protocol for node sleep scheduling in order to maximize network lifetime while satisfying coverage constraints. They also find that decreasing the detection delay also decreases the lifetime, but show that their optimized schedule, where nodes calculate their own wake-up times based on the schedules of neighboring nodes, performs better that either synchronized or random transitions between the two modes. However, none of these consider battery recovery in their models. Niyato, Hossain, and Fallahi (2007) analyze and compare different sleep/wake strategies for solar-powered nodes to optimize node lifetime, with a battery model that allows recovery, but do not consider network behavior. Their assumptions include that energy consumption occurs at a constant rate and the time/energy spent on switching between modes is negligible and therefore can be ignored. Jongerden et al. (2010) consider the parameter settings for maximizing the battery lifetime, instead of network lifetime. They find that by using the recovery effect to gain more power for the battery has a significant effect on the lifetime. They also find that there is a natural bound on the lifetime that no scheduling protocol can surpass.

\subsection{Möbius Modeling Tool}

The modeling research conducted for this research was done using the Möbius (Deavours et al. 2002) modeling framework. Möbius was developed to integrate several modeling formalisms, as well as multiple solvers. It can support Stochastic Activity Networks (SANs), Performance Evaluation Process Algebras (PEPAs), Stochastic Petri Nets (SPNs), Markov processes and queuing networks, and allows a model to be composed of all the different types. It offers both simulation and numerical analysis of Markov processes. Submodels can be composed into larger models, with the ability to share data between submodels. We expand further on the terminology of Möbius, since this is the framework chosen for the research presented here.

In Möbius, the base unit is an atomic model. As a simple example, we consider a basic two-state node with two state variables, Awake and Sleep, and two states, $\mu_{A}$ and $\mu_{S}$, that indicate if the system is up $\left(\mu_{A}(\mathrm{~A}, \mathrm{~S})=(1,0)\right)$ or down $\left(\mu_{S}(\mathrm{~A}, \mathrm{~S})=(0,1)\right)$. There are two events, going to sleep (ToSleep) and 
(a)

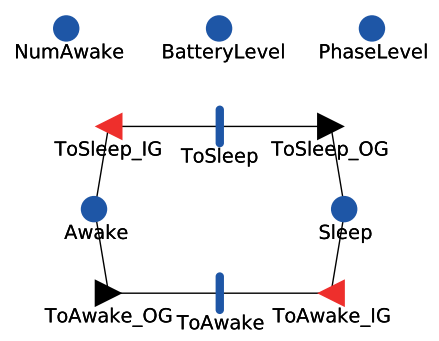

(b)

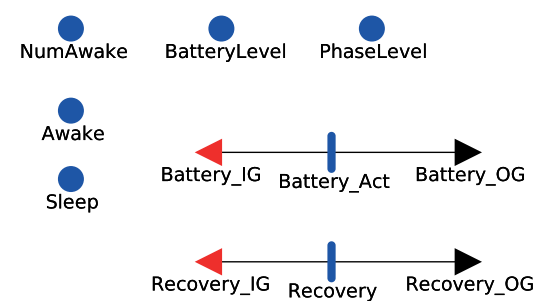

(c)

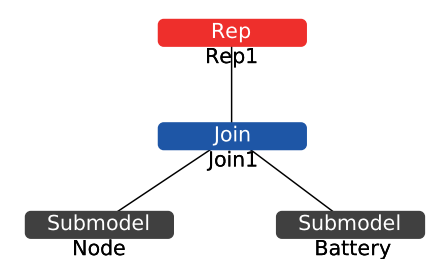

Figure 1: The Möbius atomic model of (a) a single sensor node, and (b) the battery, with (c) the Möbius composed model of multiple nodes under a Rep/Join composition.

waking up (ToAwake), where ToSleep can only occur in state $\mu_{A}$ (the unit is up) and ToAwake can only occur in state $\mu_{S}$ (the unit is down). Where these events can occur, we have a sleep transition rate of $\alpha_{S}$, a wake transition rate of $\alpha_{A}$, with $\alpha_{A}, \alpha_{S}>0$, and the transition value to be the opposite state. That is, in state $\mu_{A}$, the node transitions to the sleep state with rate $\alpha_{S}$, and the new state becomes $\mu_{S}$.

An atomic model within the SAN formalism used in Möbius is shown in Figure 1(a). In the SAN, the place primitive, pictured as a solid circle, represents the state of the model as an integer that can be modified as the state changes. For instance, the place Awake represents whether a unit is working (value equals 1) or not (value equals 0 ), with the opposite values for the place Sleep. A third state variable, NumAwake, is used by the full system to share the number of nodes, or submodels, in the system that are in the Awake state with all nodes in the system. It should be noted that in state $\mu_{A}, \mu_{A}$ (NumAwake) has values between 1 and $n$ (where $n$ is the number of submodels in the system) while in state $\mu_{S}, \mu_{S}$ (NumAwake) has values between 0 and $n-1$. Places can be of more complex data types such as user-defined structures or arrays in addition to primitive types like integer and floating point. The other places in the model are used in connection with the battery model, which is discussed later.

The transitions between states are controlled by the activity primitive, shown as a thick vertical line. Transition rates can be the rate of an exponential distribution that models the time the node stays in a state, but Möbius supports a variety of distributions that a modeler can chose from to describe time delays. Another SAN primitive, output gates, depicted by the right arrows, allows the modeler to also specify complex state changes, described through $\mathrm{C}++$ code. In the example above, the transition from state Awake to Sleep performs a decrement of Awake, increment of Sleep, and decrement of NumAwake. All submodels in the full system of the same type will share the place NumAwake since they will all contribute to how many of the submodels are in the Awake state. These places can also be used to calculate measures of interest (e.g., how often and for how long a submodel is in the sleep mode). The final SAN primitive, input gates, depicted by the left arrows, allows conditions to be set for when an activity can fire. In this model, they are used to condition the transitions to occur such that the desired coverage percentage is preserved, as well as considering the state of the battery, as discussed later.

For models with a large number of places and transitions, it is useful to apply some structuring mechanism or composition operations for SANs. Here, we use a composition operation to construct a larger model with $n$ submodels. Figure 1(c) shows how we make use of the Möbius Rep/Join composition, which provides a composition operator, Rep, to instantiate $n$ copies of the submodel to build an aggregate model. The other composition operator, Join, connects two submodels without replication. All instances can share a user-given set of state variables/places, (e.g., NumAwake). We could then build this into a larger model through the use of another Rep or Join operator. Alternative composition methods are so called action synchronization and graph composition, see (Deavours et al. 2002) for details.

In Möbius, measures of interest (such as the probability of the submodel being in the Awake state) are defined through reward variables. We can define a performance variable, numAwakeProb, to return the value of the place NumAwake at either a specific moment in time (transient analysis) or on average (steady 


\section{Lamprecht and Kemper}

state analysis). Once the model is fully defined through the atomic model, composed model, and reward measurements, Möbius allows a modeler to solve for the reward measurements either through numerical analysis, with the accompanying state space generation, or discrete-event simulation. More information about all of these procedures and formalisms can be found in (Deavours et al. 2002) or through the program website (PERFORM 2011).

\section{MODEL DESCRIPTION}

The goal of this study is to examine the influence of the inclusion of various aspects of the battery into the model on the overall system behavior. We specifically consider the constraint of network coverage and the performance measure of network lifetime. We use a simple sensor node with the two modes as previously described, modeled in a network of 100 nodes.

\subsection{Sensor Node}

For this research, we expand the node introduced in the previous section to include the battery model to follow what is used in Ren et al. (2005). For the experiments done here, a node has a period for its Awake-Sleep cycle with an active_ratio for the fraction of the period spent in the Awake mode. Thus, the transition rate from the Awake mode is period $*$ active_ratio, which is modeled with an exponential distribution with a mean of the rate inverse and the transition rate from the Sleep mode is period $*(\mathbf{1}$ - active_ratio), again modeled with an exponential distribution with a mean of the inverse of the rate. The exponential distribution is chosen because it guarantees that the rates will be positive and it allows numerical analysis to be done on the associated Continuous Time Markov Chain, if desired. The exponential distribution is a simple non-negative distribution In addition to modeling the transitions from a S leep mode to an Awake mode, we also want to model the battery level. The battery model is shown in Figure 1(b). The place BatteryLevel holds the battery level, which is depleted only when the node is in the Awake mode (controlled by the input gate Battery_IG) at a rate of energy_rate (controlled by the activity Battery_Act). This means that given an initial battery level of max_battery, if the node never goes to sleep, the battery will be depleted in max_battery / energy_rate time units. This time is extended by the amount of time the node spends in the sleep mode. The output gate Battery_OG ensures that when the battery is depleted, the node automatically goes to the Sleep mode. In the node model, the transition from $\mathrm{S}$ leep to Awake is conditioned first on the battery level being greater than 0 (controlled by the input gate ToAwake_IG), so a fully depleted battery becomes an absorbing state. Once all nodes have reached this state, the system is in a final, failed state. The transition from Awake to Sleep is conditioned on the requirement that at least the coverage percentage of nodes remains awake (controlled by the input gate ToSleep_IG). That is, if the coverage requirement is not met, then the node remains awake for another time period (as defined by the transition rate). Similarly, the transition from Sleep to Awake is further conditioned on not needing more than the coverage percentage of nodes awake (controlled by the input gate ToAwake_IG). That is, if the coverage requirement is met, the node will remain in the sleep mode. The place Phaselevel tracks the phase of the node, which relates to the time the node spends in the Awake mode.

Jongerden et al. (2010) show that when modeling batteries, including the recovery effect, or the phenomenon that batteries recharge when not in use or when in low usage, affects the lifetime of a battery. According to Jongerden et al. (2010), the simplest recovery model is to allow a constant recovery effect, but further detail can be added to reflect the fact that the weaker the battery, the less recovery occurs. In the model used here, the recovery activity and gates control battery recovery, which can only occur in the sleep mode if the battery has not been fully depleted. That is, the input gate Recovery_IG puts the condition on the Recovery activity so the activity is only enabled when Sleep equals 1 and BatteryLevel is not zero, since power is needed to make power, and BatteryLevel is less than the maximum charge, since this is the limit on the amount of power that the battery can hold. The recovery rate is also an 


\section{Lamprecht and Kemper}

Table 1: Average network lifetime for nodes with no battery recovery, simple battery recovery, and phase-based battery recovery for increasing required coverage percentages.

\begin{tabular}{|c||c|c|c|}
\hline Cov. \% & No & Simple & Phase \\
\hline \hline 60 & 64.22 & 65.58 & 76.76 \\
\hline 70 & 58.93 & 60.02 & 68.36 \\
\hline 80 & 54.27 & 54.73 & 60.76 \\
\hline 90 & 50.43 & 50.52 & 54.04 \\
\hline 100 & 46.08 & 45.92 & 46.49 \\
\hline
\end{tabular}

exponential distribution with either the constant mean or with the state-dependent value. Both the phase level and battery recovery aspects of the model are used in later experiments.

Since the node and the battery models are defined in separate atomic models, to form a complete node we must join the two parts of the unit. In connecting a node model and a battery model through the Join operator, all places in each model are shared since this is information both parts of the unit need to access and change. For instance, when the battery activity fires, the BatteryLevel must be decremented, but if that puts the level at 0 , the battery has died, so the node must be put to sleep and the total number of nodes that are awake must also be decremented, so the places Awake, NumAwake, and Sleep are all given new values.

\subsection{Sensor Network}

The simplest way to specify the network is with the Rep/Join operator as in Figure 1(c). The Join operator connects the two parts of the node as described above. The Rep operator then holds the information on the number instances of sensor nodes. For an initial state of the network, we have chosen to compare when all nodes start in the Awake mode, all nodes start in the Sleep mode, or having exactly the coverage percentage of the nodes start in the Awake mode. This simple model ignores spatial aspects, ignores communication and assumes that the only dependence across nodes is the current level of coverage.

\section{RESULTS}

The following results are calculated through simulation, run 1000 times to gain a $95 \%$ confidence interval. The maximum battery level for each node set at 30 with an energy_rate of 1 , while the period is 1.1 with an active_ratio of 0.5 .

\subsection{Coverage Percentage under Different Initial Conditions}

Figure 2 show the network lifetime for the various starting conditions. First, Figure 2(a) shows results from setting all nodes to start in the Awake mode, while Figure 2(b) comes from experiments where all nodes start in the Sleep mode. We also tested the initial condition where some nodes start in the Awake and the rest in the Sleep mode, based on the desired coverage percentage. In the first two cases, it can be seen that the network lifetime is the same regardless of the initial condition of the nodes in the network. The results for the mixed starting mode are not included here to reduce the redundant figures. Since the initial state of the network does not affect the overall network affect, for all future simulations, we use the initial state of all nodes being in the Awake mode. In Table 1, the first column lists the expected value for the lifetime of the network for the chosen coverage percentages with no battery recovery. We use these as base values for comparison to the models that include more information. 
Lamprecht and Kemper

(a)

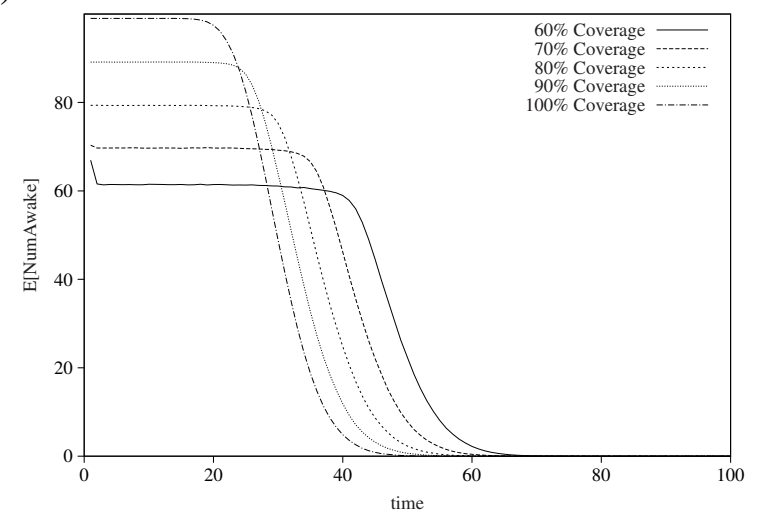

(b)

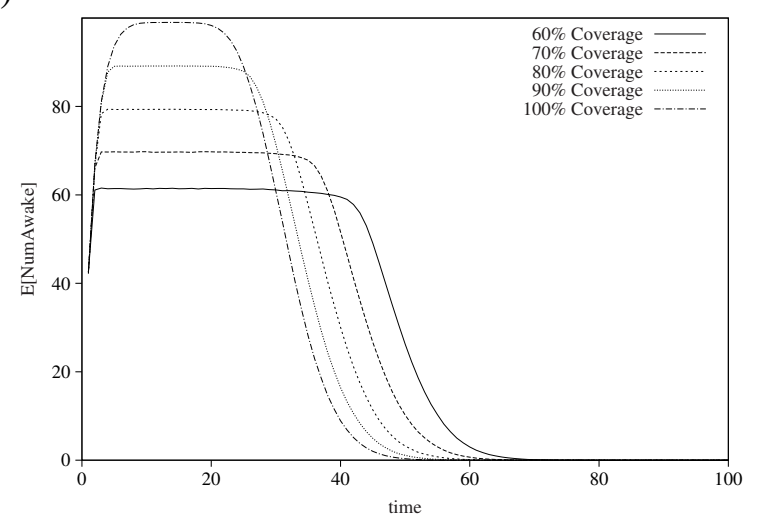

Figure 2: Expected number of nodes in the Awake mode given the starting condition that all nodes start in (a) the Awake mode or (b) the Sleep mode.

\subsection{Coverage Percentage with Battery Recovery}

Figure 3(a) shows the results for experiments on a network of nodes that incorporate battery recovery, measured by the expected number of nodes in the Awake mode. The results shown are for the simplest recovery effect, which shows a slight increase in the network lifetime. The second column of Table 1 lists the expected network lifetime for the chosen coverage percentages with this simple recovery. The increase in the network lifetime is less than $2 \%$ over the model with no recovery, so the inclusion of a simple battery recovery model does not change the outcome of the network study. One point of interest that can be seen in these results, and all others, is that with a higher required coverage percentage, more nodes will spend all or nearly all of their time in the Awake mode, so there is little or no recovery and the lifetime of the network is dependent on the energy level of a node.

Figure 3(b) shows the results for experiments that incorporate a more realistic battery recovery. As before, battery recovery occurs only when the node is in the Sleep mode, but the amount of recovery is dependent on the phase of the node $p$, and the current battery level, $b$. The phase of a node is defined by the amount of time spent in the Awake mode, as measured by the energy spent by the battery. Thus, as the battery is depleted, the phase increases. When the battery is nearly full, the phase level will be low. The longer a node remains in the Awake mode, the more the battery is depleted and the higher the phase level. Once the node goes to the Sleep mode, the phase and battery levels determine the recovery amount, $r$, as defined by (Chiasserini and Rao 2001):

$$
r=e^{g_{N}(N-b)-g_{C}(p)}
$$

where $N$ is the maximum battery level and $g_{N}$ and $g_{C}$ are parameters dependent on the recovery properties of the battery. The expected lifetime of the network for the chosen percentages are listed in the third column of Table 1. For coverage percentage of 80 , there is a $13 \%$ change in the calculated network lifetime, which shows that this simple inclusion can have a large impact on the study results.

\subsection{Coverage Percentage with Battery Recovery and Modified Sleep Schedule}

Modeling with the more refined battery model introduces the desire to schedule the transition to the Sleep mode based on the time spent in the Awake mode. That is, the longer a node stays awake, the more likely it is to transition. Therefore, we condition the Awake-Sleep transition on either the coverage percentage being met (as what has been done for the above results) or by the phase level reaching a specified maximum value. Given a maximum battery level of 30 , we examine the network behavior for a maximum phase level of 5,10,15, and 20. Higher phase level results are not included here because when the maximum phase 
(a)

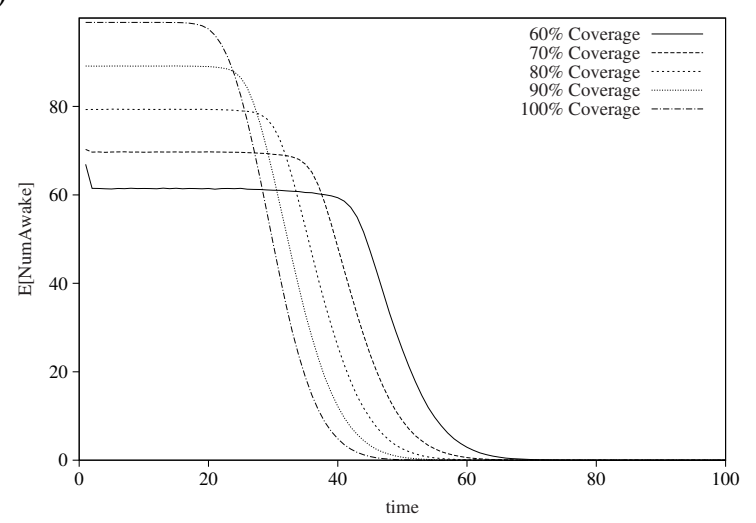

(b)

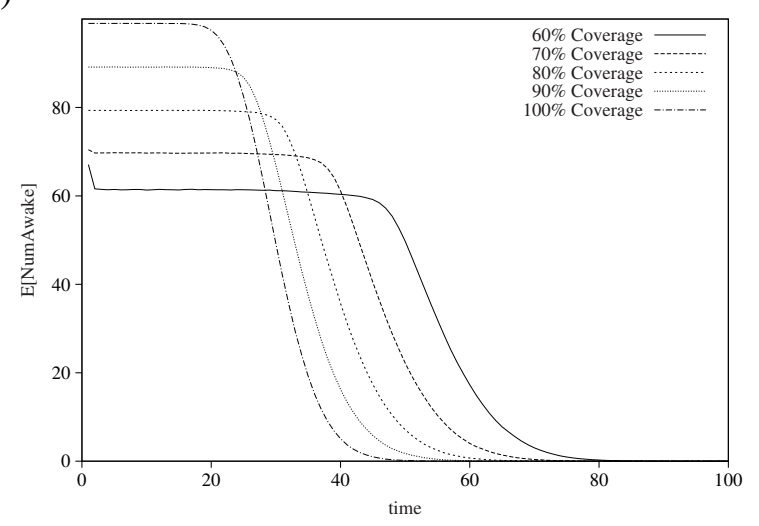

Figure 3: Expected number of nodes in the Awake mode given the starting condition that all nodes start in the Awake mode and allowing battery recovery during the Sleep mode (a) at a constant rate (simple recovery) or (b) at a rate dependent on the phase and battery level.

Table 2: Average network lifetime for nodes with phase-based battery recovery and phase-driven sleep schedule for various maximum phase levels and increasing required coverage percentages.

\begin{tabular}{|c|c|c|c|c|}
\hline Cov. \% & Max = 5 & Max = 10 & Max = 15 & Max = 20 \\
\hline 60 & 93.55 & 87.72 & 85.33 & 84.83 \\
\hline 70 & 82.59 & 75.92 & 74.29 & 73.31 \\
\hline 80 & 72.31 & 65.76 & 64.22 & 63.69 \\
\hline 90 & 68.85 & 57.63 & 55.66 & 55.94 \\
\hline 100 & 68.05 & 55.11 & 50.29 & 49.51 \\
\hline
\end{tabular}

level nears the maximum battery level, the network lifetime values are within $2 \%$ of each other. Figure 4 shows the network behavior through the expected number of awake nodes under the chosen coverage percentages, while Table 2 lists the expected network lifetime for the other four maximum phase levels examined.. Maintaining a low phase-level, or shortening the amount of time spent in the Awake mode, which allows for greater battery recovery, increases the expected network lifetime by nearly $40 \%$ over the simple recovery model. This is because with a low maximum phase, more state switching is required, which means more time is spent in the Sleep mode, which means more recovery time. So, we find again, that a simple inclusion of additional information in the battery model can have a large impact on the study results. We also find that with a high coverage percentage $(>90 \%)$, nodes will quickly reach maximum phase level, and so we see in the results a quick drop in the expected number of nodes in the Awake mode. However, the system then tries to increase the number of Awake nodes to meet the percentage requirements, so we see a brief rise in this number, until nodes begin to lose all power, the recovery amount becomes smaller, and the network descends to a failed state.

\subsection{Coverage Percentage with Greater Variance}

Figure 5 shows the results for experiments on modifying the variance of the transitions between the Awake and Sleep modes on the expected number of nodes in the Awake mode. High variance in a sensor network becomes interesting when there are unreliable links between the sensors (Halgamuge et al. 2009). The variance for the exponential distribution is 3.3, with a mean of 1.8181. To increase the variance in the transition rates between the two modes, a hyper-exponential distribution was used to increase the variance by factors of approximately 100 and 1000, which leads to a high index of dispersion. For both variance 
(a)

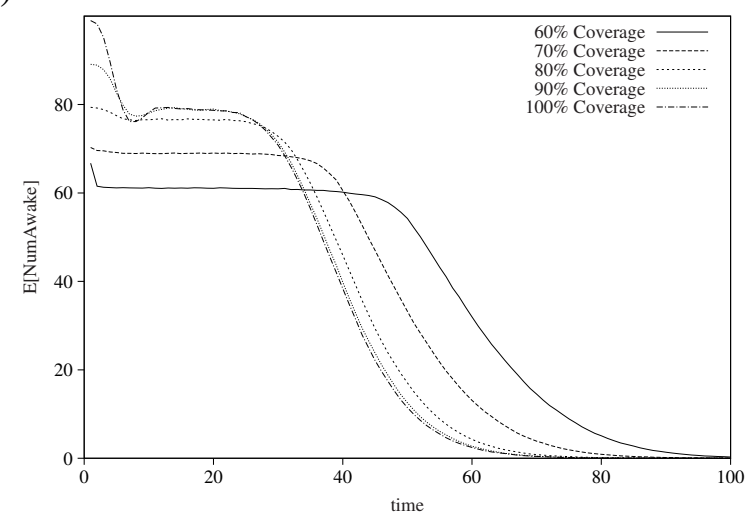

(c)

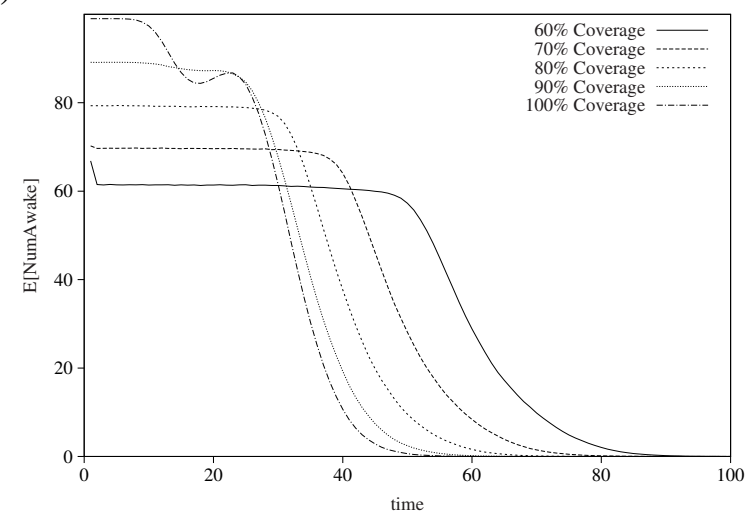

(b)

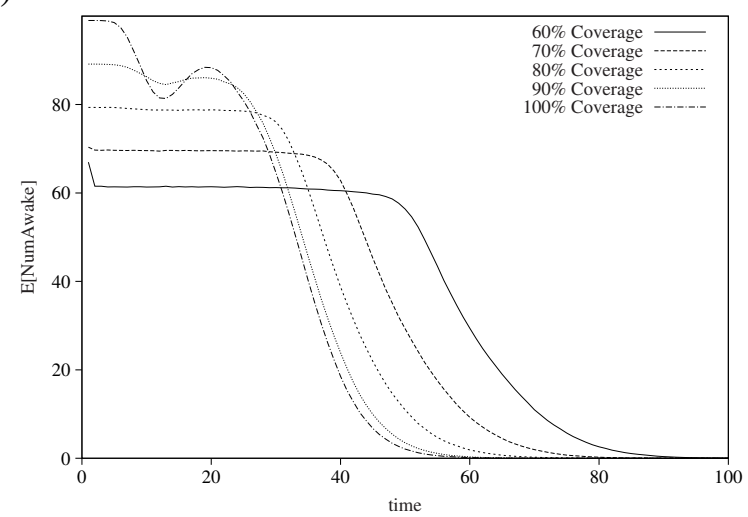

(d)

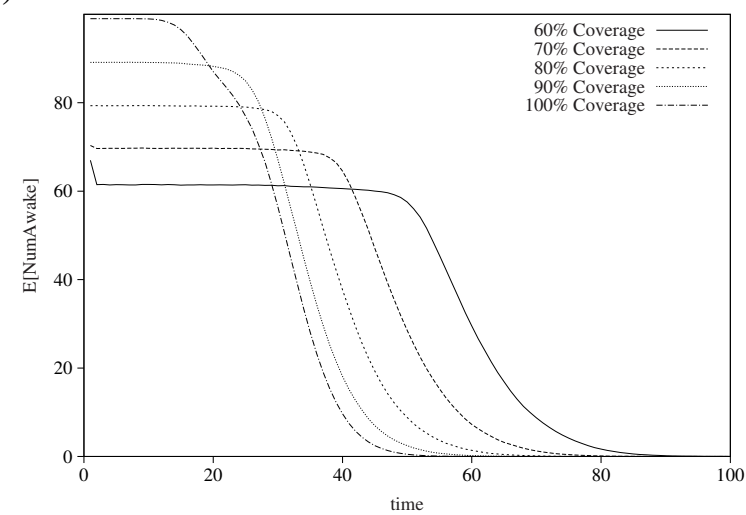

Figure 4: Expected number of nodes in the Awake mode given the starting condition that all nodes start in the Awake mode, allowing battery recovery during the Sleep mode, and scheduling sleep when the phase level reaches (a) 5 or (b) 10 or (c) 15 or (d) 20). 
Lamprecht and Kemper

(a)

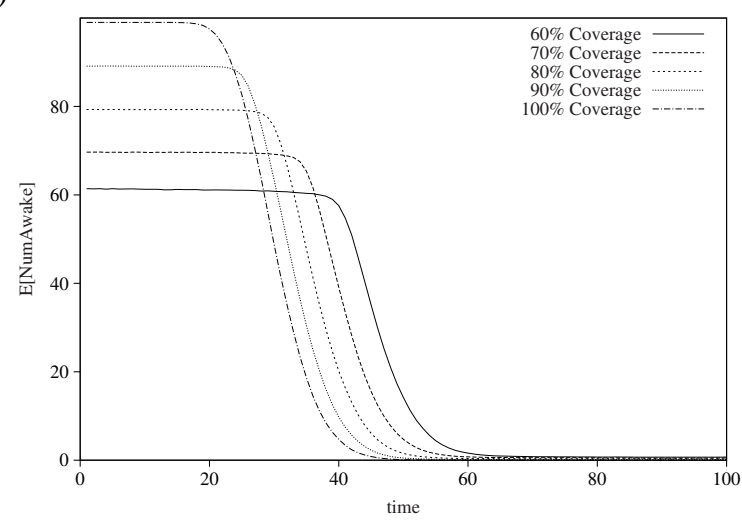

(b)

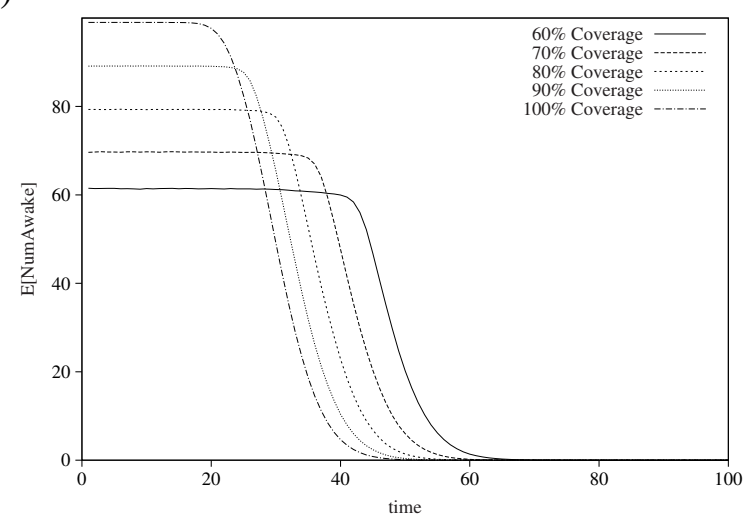

Figure 5: Expected number of nodes in the Awake mode given the starting condition that all nodes start in the Awake mode and allowing greater variance, (a) times 100 and (b) times 1000, on the mean time to sleep.

changes, the behavior is similar to the original experiments. This suggests that even under extreme variance, the network is stable in its behavior.

\section{CONCLUSIONS}

The objective of this paper was to explore the sensitivity of the network lifetime of a wireless sensor network to the inclusion of different modeling aspects. By combining the different parts of a WSN, and comparing the expected network lifetime, we conclude that simple model behaviors can be included and have a large impact on the network lifetime. Specifically, when modeling a WSN, while a common assumption is to use a simple battery model that has only energy depletion, the inclusion of battery recovery can lead to as much as a $40 \%$ increase in the expected network lifetime. Another observation we have made is that there seems to be two phases to the network behavior. The first phase is while the required coverage percentage can be met and the second phase is the time while the percentage cannot be met, but not all nodes have completely lost power. This is where the impact of the different kinds of models can be seen best. The length of the first phase is dependent on both the coverage percentage and the recovery that can occur while the second phase is dependent on the amount of energy the nodes have left once some nodes begin to die, which is also dependent on the ability of the nodes to have energy recovery during the first phase.

\section{REFERENCES}

Ailamaki, A., C. Faloutos, P. S. Fischbeck, M. J. Small, and J. VanBriesen. 2003, December. "An environmental sensor network to determine drinking water quality and security". SIGMOD Record 32:47-52.

Akyildiz, I. F., D. Pompili, and T. Melodia. 2005. "Underwater acoustic sensor networks: research challenges". Ad Hoc Networks 3 (3): 257 - 279.

Arora, A., P. Dutta, S. Bapat, V. Kulathumani, H. Zhang, V. Naik, V. Mittal, H. Cao, M. Demirbas, M. Gouda, Y. Choi, T. Herman, S. Kulkarni, U. Arumugam, M. Nesterenko, A. Vora, and M. Miyashita. 2004. "A line in the sand: a wireless sensor network for target detection, classification, and tracking". Computer Networks 46 (5): 605 - 634. Military Communications Systems and Technologies.

Cao, Q., T. Abdelzaher, T. He, and J. Stankovic. 2005. "Towards optimal sleep scheduling in sensor networks for rare-event detection". In Proceedings of the 4th International Symposium on Information Processing in Sensor Networks, IPSN '05: IEEE Press.

Chiasserini, C.-F., and M. Garetto. 2004. "Modeling the performance of wireless sensor networks". IEEE INFOCOM 1:231-242. 


\section{Lamprecht and Kemper}

Chiasserini, C.-F., and R. R. Rao. 2001, July. "Energy efficient battery management". IEEE Journal on Selected Areas in Communications 19 (7): 1235 -1245.

Deavours, D. D., G. Clark, T. Courtney, D. Daly, S. Derisavi, J. M. Doyle, W. H. Sanders, and P. G. Webster. 2002. "The Möbius framework and its implementation". IEEE Transactions on Software Engineering 28 (10): 956-969.

Douglas, C. C., R. A. Lodder, R. E. Ewing, Y. Efendiev, G. Qin, J. Coen, M. Kritz, J. D. Beezley, J. Mandel, M. Iskandarani, A. Vodacek, and G. Haase. 2006, December. "DDDAS approaches to wildland fire modeling and contaminant tracking". In Proceedings of the 2006 Winter Simulation Conference, edited by L. F. Perrone, F. P. Wieland, J. Liu, B. G. Lawson, D. M. Nicol, and R. M. Fujimoto, WSC '06, 2117-2124. Piscataway, New Jersey: Institute of Electrical and Electronics Engineers, Inc.

Halgamuge, M. N., T.-K. Chan, and P. Mendis. 2009. "Experimental study of link quality distribution in sensor network deployment for building environment". EJSE Special Issue: Sensor Network on Building Monitoring: from theory to real application.

Jongerden, M., A. Mereacre, H. Bohnenkamp, B. Haverkort, and J.-P. Katoen. 2010, August. "Computing optimal schedules of battery usage in embedded systems". IEEE Transactions on Industrial Informatics 6 (3): 276-286.

Law, A. M., and W. D. Kelton. 2000. Simulation Modeling and Analysis. McGraw-Hill Higher Education.

Lu, M., J. Wu, M. Cardei, and M. Li. 2009, April. "Energy-efficient connected coverage of discrete targets in wireless sensor networks". International Journal of Ad Hoc and Ubiquitous Computing 4:137-147.

Niyato, D., E. Hossain, and A. Fallahi. 2007, February. "Sleep and wakeup strategies in solar-powered wireless sensor/mesh networks: performance analysis and optimization". IEEE Transactions on Mobile Computing 6:221-236.

PERFORM 2011. "The Möbius tool”. www.mobius.uiuc.edu.

Ren, S., Q. Li, H. Wang, X. Chen, and X. Zhang. 2005. "Analyzing object detection quality under probabilistic coverage in sensor networks". In Quality of Service IWQoS 2005, edited by H. de Meer and N. Bhatti, Volume 3552 of Lecture Notes in Computer Science, 107-122. Springer Berlin / Heidelberg.

$\mathrm{Xu}$, N., S. Rangwala, K. K. Chintalapudi, D. Ganesan, A. Broad, R. Govindan, and D. Estrin. 2004. "A wireless sensor network for structural monitoring". In Proceedings of the 2nd International Conference on Embedded Networked Sensor Systems, SenSys '04, 13-24.

\section{AUTHOR BIOGRAPHIES}

RUTH LAMPRECHT is an Ph.D. candidate in Computer Science at the College of William and Mary and an instructor at Virginia Union University in the Department of Computer Information Science / Computer Science. Her research interests include simulation, numerical analysis, and optimization. Her web page can be found via http://www.cs.wm.edu/ rlampy and her email address is rlampy@cs.wm.edu.

PETER KEMPER is an Associate Professor in the Department of Computer Science at the College of William and Mary (previously TU Dortmund and TU Dresden, Germany). His research interests include modeling techniques and tools for performance, performability and dependability analysis of systems. He contributed to analysis techniques for the numerical analysis of Markov chains, model checking stochastic models, techniques for simulation optimization. His web page can be found via http://www.cs.wm.edu/ kemper and his email address is kemper@cs.wm.edu. 